\title{
Perfect Local Computability and Computable Simulations
}

\author{
Russell Miller ${ }^{1}$ and Dustin Mulcahey ${ }^{2}$ \\ 1 Queens College of CUNY \\ 65-30 Kissena Blvd., Flushing, NY 11367 USA \\ and \\ The CUNY Graduate Center \\ Russell.Miller@qc. cuny.edu \\ http://qcpages.qc. cuny. edu/ rmiller \\ 2 The CUNY Graduate Center \\ 365 Fifth Avenue, New York NY 10016 USA \\ dustinmulcahey@gmail.com \\ https://wfs.gc. cuny.edu/DMulcahey/www/
}

\begin{abstract}
We study perfectly locally computable structures, which are (possibly uncountable) structures $\mathcal{S}$ that have highly effective presentations of their local properties. We show that every such $\mathcal{S}$ can be simulated, in a strong sense and even over arbitrary finite parameter sets, by a computable structure. We also study the category theory of a perfect cover of $\mathcal{S}$, examining its connections to the category of all finitely generated substructures of $\mathcal{S}$.
\end{abstract}

Key words: Category theory, computability, local computability, perfect local computability, simulation

\section{Introduction}

Locally computable structures were introduced in [3], as a method for effective presentation of uncountable structures. One considers a structure $\mathcal{S}$ locally rather than globally, giving presentations of all finitely generated substructures of $\mathcal{S}$, up to isomorphism, along with a description of the ways in which these "pieces" of $\mathcal{S}$ fit together to form the entire structure. We review these definitions below. The entire package, including both the pieces and the ways they fit together, comprise a cover of $\mathcal{S}$, which is said to be uniformly computable if it can be given in a sufficiently effective manner.

The notion of a perfect cover was also defined in [3], and it is stated there (and proven in [4]) that a countable structure has a perfect cover iff it is computably presentable. This suggests that for uncountable structures, perfect local computability (i.e. having a perfect cover) is a reasonable analogue for computable presentability. In this paper we further explore perfectly locally computable structures, showing that all such structures have computable simulations, or computably presentable elementary substructures realizing the same types. 
Such substructures may be used in natural ways to simulate operations in the larger structure - which in general is not countable, hence not itself computably presentable.

A cover bears a natural resemblance to a category, and indeed is closely related to the category $\mathbf{F G S u b}(\mathcal{S})$ of all finitely generated substructures of $\mathcal{S}$ under inclusion maps. Of course, $\mathbf{F G S u b}(\mathcal{S})$ will be uncountable if $\mathcal{S}$ is, whereas uniformly computable covers are always countable. Moreover, Definition 22 does not require that the morphisms among objects (i.e. the embeddings of one finitely generated substructure into another) be closed under composition. However, for the specific case of a perfect cover, we may assume closure under composition, and therefore we find ourselves working in a (computable) category. We examine the category-theoretic properties of such covers in Section 4.

Our computability-theoretic terminology coincides with that of [5], the standard reference for the subject, and the definitions in local computability from [3] are used here without alteration. For category theory, we recommend [2].

\section{Local Computability Definitions}

Let $T$ be a $\forall$-axiomatizable theory in a signature of size $n<\omega$. (The theory of fields is a good example to keep in mind, skolemized to have function symbols for negation and inversion.) We first consider simple covers of a model $\mathcal{S}$ of $T$. These describe only the finitely generated substructures of $\mathcal{S}$, with no attention paid to any relations between those substructures.

Definition 21 A simple cover of $\mathcal{S}$ is a (finite or countable) collection $\mathfrak{A}$ of finitely generated models $\mathcal{A}_{0}, \mathcal{A}_{1}, \ldots$ of $T$, such that:

- every finitely generated substructure of $\mathcal{S}$ is isomorphic to some $\mathcal{A}_{i} \in \mathfrak{A}$; and

- every $\mathcal{A}_{i} \in \mathfrak{A}$ embeds isomorphically into $\mathcal{S}$.

This simple cover is uniformly computable if every $\mathcal{A}_{i} \in \mathfrak{A}$ is a computable structure and the sequence $\left\langle\left(\mathcal{A}_{i}, \bar{a}_{i}\right)\right\rangle_{i \in \omega}$ can be given uniformly: there must exist a single computable function which, on input $i$, outputs a tuple of elements $\left\langle e_{1}, \ldots, e_{n},\left\langle a_{0}, \ldots, a_{k_{i}}\right\rangle\right\rangle \in \omega^{n} \times \mathcal{A}_{i}^{<\omega}$ such that $\left\{a_{0}, \ldots, a_{k_{i}}\right\}$ generates $\mathcal{A}_{i}$ and $\varphi_{e_{j}}$ computes the $j$-th function, constant, or relation in $\mathcal{A}_{i}$.

Definition 22 A cover of $\mathcal{S}$ consists of a simple cover $\mathfrak{A}=\left\{\mathcal{A}_{0}, \mathcal{A}_{1}, \ldots\right\}$ of $\mathcal{S}$, along with sets $I_{i j}^{\mathfrak{A}}$ (for all $\mathcal{A}_{i}, \mathcal{A}_{j} \in \mathfrak{A}$ ) of injective homomorphisms $f: \mathcal{A}_{i} \hookrightarrow \mathcal{A}_{j}$, such that:

- for all finitely generated substructures $\mathcal{B} \subseteq \mathcal{C}$ of $\mathcal{S}$, there exist $i, j \in \omega$ and $f \in I_{i j}^{\mathfrak{A}}$ and isomorphisms $\beta: \mathcal{A}_{i} \rightarrow \mathcal{B}$ and $\gamma: \mathcal{A}_{j} \rightarrow \mathcal{C}$ with $\beta=\gamma \circ f$; and

- for every $i$ and $j$ and every $f \in I_{i j}^{\mathfrak{A}}$, there exist substructures $\mathcal{B} \subseteq \mathcal{C}$ of $\mathcal{S}$ and isomorphisms $\beta: \mathcal{A}_{i} \rightarrow \mathcal{B}$ and $\gamma: \mathcal{A}_{j} \rightarrow \mathcal{C}$ with $\beta=\gamma \circ f$.

This cover is uniformly computable if $\mathfrak{A}$ is a uniformly computable simple cover of $\mathcal{S}$ and there exists a c.e. set $W$ such that for all $i, j \in \omega$,

$$
I_{i j}^{\mathfrak{A}}=\left\{\varphi_{e} \mid \mathcal{A}_{i}:\langle i, j, e\rangle \in W\right\} .
$$


A structure $\mathcal{B}$ is locally computable if it has a uniformly computable cover.

We will be concerned with a particularly nice class of locally computable structures, so nice that we call them perfectly locally computable. Here we have much stronger connections between the substructures of $\mathcal{S}$ and the structures $\mathcal{A}_{i}$ in our effective description.

Definition 23 Let $\mathfrak{A}$ be a uniformly computable cover for a structure $\mathcal{S}$. A set $M$ is a correspondence system for $\mathfrak{A}$ and $\mathcal{S}$ if it satisfies the following five rules:

1. Each element of $M$ is an embedding of some $\mathcal{A}_{i} \in \mathfrak{A}$ into $\mathcal{S}$; and

2. For every $\mathcal{A}_{i} \in \mathfrak{A}$, there exists a $\beta \in M$ with domain $\mathcal{A}_{i}$; and

3. For every finitely generated substructure $\mathcal{B}$ of $\mathcal{S}$, there exists a $\beta \in M$ with image $\mathcal{B}$; and

4. For every $\mathcal{A}_{i} \in \mathfrak{A}$, every $\beta \in M$ with domain $\mathcal{A}_{i}$, every $\mathcal{A}_{j} \in \mathfrak{A}$, and every $f \in I_{i j}^{\mathfrak{A}}$, there exists a $\gamma \in M$ with domain $\mathcal{A}_{j}$ such that $\beta=\gamma \circ f$ (and hence $\left.\beta\left(\mathcal{A}_{i}\right) \subseteq \gamma\left(\mathcal{A}_{j}\right)\right)$; and

5. For every $\mathcal{A}_{i} \in \mathfrak{A}$, every $\beta \in M$ with domain $\mathcal{A}_{i}$, and every finitely generated $\mathcal{B} \subseteq \mathcal{S}$ such that $\beta\left(\mathcal{A}_{i}\right) \subseteq \mathcal{B}$, there exists an $\mathcal{A}_{j} \in \mathfrak{A}$, a $\gamma \in M$ with domain $\mathcal{A}_{j}$ and image $\mathcal{B}$, and an $f \in I_{i j}^{\mathfrak{A}}$ such that $\beta=\gamma \circ f$.

A correspondence system is perfect if it also satisfies:

6. For every finitely generated $\mathcal{B} \subseteq \mathcal{S}$, if $\beta: \mathcal{A}_{i} \rightarrow \mathcal{B}$ and $\gamma: \mathcal{A}_{j} \rightarrow \mathcal{B}$ both lie in $M$, then $\gamma^{-1} \circ \beta \in I_{i j}^{\mathfrak{A}}$.

If a perfect correspondence system exists, then its elements are called perfect matches between their domains and their images. The uniformly computable cover $\mathfrak{A}$ is then called a perfect cover for $\mathcal{S}$, and $\mathcal{S}$ itself is said to be perfectly locally computable.

For example, it is proven in [3] that every algebraically closed field is perfectly locally computable. The field of real numbers, on the other hand, is not perfectly locally computable, and the ordered field of real numbers is not even locally computable.

Other uncountable examples are known. In the language of linear orders, Cantor space is perfectly locally computable. Likewise, if Cantor space is viewed as the top level of the tree $2^{<\omega+1}$ in the language of partial orders - with a additional unary predicate $C$ identifying that top level, and also with an immediatepredecessor function on the elements of all lengths strictly between 0 and $\omega$, if one wishes - then this entire tree, under the additional predicate and function symbol, is perfectly locally computable. However, if one adds the linear order on the top level of that tree, then the new structure loses its perfect local computability, even though both of the structures merged together had it; in fact the new structure is barely even locally computable.

Another theorem from [3] and [4] shows that perfect local computability can be viewed as a natural extension of the notion of computability - or more precisely, computable presentability - for countable structures. 
Theorem 24 Let $\mathcal{S}$ be any countable structure. Then $\mathcal{S}$ is computably presentable iff $\mathcal{S}$ is perfectly locally computable.

In the following, we consider perfectly locally computable structures, whether countable or uncountable, and show that in a certain sense, we can dispense with their covers and indeed with the structures themselves, and replace them with structures that actually are countable and computably presented. The notion of a computable simulation will be the precise version of this substitution.

\section{Computable Simulations}

Definition 31 Let $\mathcal{S}$ be any structure. A simulation of $\mathcal{S}$ is an elementary substructure $\mathcal{B} \preceq \mathcal{S}$ such that $\mathcal{B}$ and $\mathcal{S}$ realize exactly the same finitary types. We often refer to any $\mathcal{A}$ isomorphic to such a $\mathcal{B}$ as a simulation of $\mathcal{S}$, even if $\mathcal{A}$ is not itself a substructure of $\mathcal{S}$. Hence a computable simulation of $\mathcal{S}$ is a computable structure isomorphic to a simulation of $\mathcal{S}$.

Lemma 1. Let $\mathcal{S}$ be locally computable, with a correspondence system $N$ over a uniformly computable cover $\mathfrak{A}$. Then $\mathcal{S}$ has a countable substructure $\mathcal{B}$ with its own correspondence system $M \subseteq N$ over $\mathfrak{A}$. If $N$ was a perfect correspondence system for $\mathcal{S}$, then $M$ is perfect for $\mathcal{B}$ as well.

Proof. $\mathcal{B}$ will be a countable union of countable substructures $\mathcal{B}_{s}$ of $\mathcal{S}$. To start, we fix for each $i \in \omega$ one map $\alpha_{i} \in N$ with domain $\mathcal{A}_{i}$, Let $M_{0}=\left\{\alpha_{i}: i \in \omega\right\}$, and let $\mathcal{B}_{0}$ be the substructure of $\mathcal{S}$ generated by the union of all the images of these $\alpha_{i}$. The conditions for a perfect cover are $\forall \exists$ conditions, so now we will be able to keep $\mathcal{B}$ countable as we close $M$ under those conditions, using the analogous conditions in the correspondence system $N$.

Assume we have defined a countable $\mathcal{B}_{s}$ and $M_{s}$. First, for every $i$, every $\alpha \in M_{s}$ with domain $\mathcal{A}_{i}$, and every $f \in I_{i j}^{\mathfrak{A}}$ (for any $j$ ), there exists some $\gamma \in N$ with domain $\mathcal{A}_{j}$ such that $f$ lifts via $\alpha$ and $\gamma$ to the inclusion $\alpha\left(\mathcal{A}_{i}\right) \subseteq \gamma\left(\mathcal{A}_{j}\right)$. Form $M_{s}^{\prime} \supseteq M_{s}$ by adjoining one such $\gamma$ to $M_{s}$ for each such $i, \alpha$ and $f$. Also, let $\mathcal{B}_{s}^{\prime}$ be generated by the union of the images of the maps in $M_{s}^{\prime}$. Clearly both $\mathcal{B}_{s}^{\prime}$ and $M_{s}^{\prime}$ remain countable.

Next, for every $i$, every $\alpha \in M_{s}$ with domain $\mathcal{A}_{i}$, and every finitely generated $\mathcal{C} \subseteq \mathcal{B}_{s}$ with $\alpha\left(\mathcal{A}_{i}\right) \subseteq \mathcal{C}$, there exists some $j$, some $f \in I_{i j}^{\mathfrak{A}}$, and some $\gamma \in N$ with domain $\mathcal{A}_{j}$ such that $f$ lifts to the inclusion $\alpha\left(\mathcal{A}_{i}\right) \subseteq \mathcal{C}$ via $\alpha$ and $\gamma$. Adjoin to $M_{s}^{\prime}$ one such $\gamma$ for each such $i, \alpha$, and $\mathcal{C}$, to form $M_{s}^{\prime \prime}$.

Finally, for every finitely generated substructure $\mathcal{C} \subseteq \mathcal{B}_{s}$, there exists a $\gamma \in N$ with image $\mathcal{C}$ (since $\mathcal{C} \subseteq \mathcal{S}$ ). Form $M_{s+1}$ by adjoining to $M_{s}^{\prime \prime}$ one such $\gamma$ for each such $\mathcal{C}$. Since $\mathcal{B}_{s}$ was countable, it has only countably many finitely generated substructures, and so $M_{s+1}$ is still countable.

It is clear that the union $\mathcal{B}=\cup_{s} \mathcal{B}_{s}$ is a countable substructure of $\mathcal{S}$, with cover $\mathfrak{A}$, and that $M=\cup_{s} M_{s}$ is a correspondence system for this $\mathcal{B}$ over $\mathfrak{A}$. Our $\mathcal{B}_{0}$ already satisfied item (2) of Definition 23, and our ensuing adjoinments satisfied (4), (5), and (3), in that order, without ever violating (1). (Of course $\mathfrak{A}$ 
is still uniformly computable as well; that definition has nothing to do with the structure covered by $\mathfrak{A}$.)

It remains to see that this $M$ is perfect for $\mathcal{B}$ whenever $N$ is perfect for $\mathcal{S}$. But this is easy: if $\alpha$ and $\gamma$ lie in $M$ and have the same image in $\mathcal{B}$, then they lie in $N$ and have the same image in $\mathcal{S}$. Since $N$ is perfect, $\gamma^{-1} \circ \alpha$ must then lie in the appropriate $I_{i j}^{\mathfrak{A}}$.

In this situation, $\mathcal{B}$ will be an elementary substructure of $\mathcal{S}$. The next lemma extends this observation. (If $\mathcal{B}$ is as in Lemma 1 , and $P$ is empty, then in the proof of Lemma 2 we may show that at every step $\psi_{s}$ is just inclusion.)

Lemma 2. Let $\mathcal{B}$ and $\mathcal{S}$ be two structures, each with a correspondence system over the same uniformly computable cover. Assume that $\mathcal{B}$ is countable. Then $\mathcal{B}$ is a simulation of $\mathcal{S}$. Indeed, for any countable set $P \subseteq \mathcal{S}$ of parameters, we can elementarily embed $\mathcal{B}$ into $\mathcal{S}$ so that its image contains $P$ and realizes the same finitary types as $\mathcal{S}$ over every finite $P_{0} \subseteq P$.

Proof. Let $\mathfrak{A}$ be a common uniformly computable cover of $\mathcal{S}$ and $\mathcal{B}$, with correspondence systems $M$ for $\mathcal{B}$ and $N$ for $\mathcal{S}$. Our embedding is built step by step, so we start by enumerating the domain of $\mathcal{B}$ as $\left\{b_{0}, b_{1}, \ldots\right\}$, and $P$ as $\left\{p_{0}, p_{1}, \ldots\right\}$. Fix an $\alpha \in M$ whose image is the substructure $\mathcal{B}_{0} \subseteq \mathcal{B}$ generated by $b_{0}$, and a $\gamma \in N$ with the same domain as $\alpha$, and define $\psi_{0}$ to be $\gamma \circ \alpha^{-1}$, with $\mathcal{B}_{0}=\operatorname{dom}\left(\psi_{0}\right) \subseteq \mathcal{B}$ and $\mathcal{C}_{0}=\operatorname{range}\left(\psi_{0}\right) \subseteq \mathcal{S}$.

At stage $t+1=2 s+1$, we extend $\psi_{t}$ so that its range contains $p_{s}$. By induction $\psi_{t}=\gamma \circ \alpha^{-1}$ for some $\gamma \in N$ and $\alpha \in M$ with common domain $\mathcal{A}_{i}$ in $\mathfrak{A}$. Let $\mathcal{C}_{t+1}$ be the substructure of $\mathcal{S}$ generated by $\mathcal{C}_{t}$ and $p_{s}$. By induction $\mathcal{C}_{t}$ is finitely generated, so there is a $\delta \in N$ with some domain $\mathcal{A}_{j} \in \mathfrak{A}$, and an $f \in I_{i j}^{\mathfrak{A}}$, such that $f$ lifts via $\gamma$ and $\delta$ to the inclusion $\mathcal{C}_{t} \subseteq \mathcal{C}_{t+1}$. In turn there is a $\beta \in M$ with domain $\mathcal{A}_{j}$ such that $f$ lifts via $\alpha$ and $\beta$ to the inclusion $\mathcal{B}_{t} \subseteq \beta\left(\mathcal{A}_{j}\right)$. Set $\mathcal{B}_{t+1}=\operatorname{range}(\beta)$ and $\psi_{t+1}=\delta \circ \beta^{-1}$.

At stage $t+1=2 s+2$, we extend the embedding $\psi_{t}$ from its current domain $\mathcal{B}_{t}$ to the structure $\mathcal{B}_{t+1}$ generated by $\mathcal{B}_{t}$ and $b_{s}$. By induction $\mathcal{B}_{t}$ is finitely generated, and $\psi_{t}=\gamma \circ \alpha^{-1}$ for some $\gamma \in N$ and $\alpha \in M$ with common domain $\mathcal{A}_{i}$ in $\mathfrak{A}$. So there is a $\beta \in M$ with some domain $\mathcal{A}_{j} \in \mathfrak{A}$, and an $f \in I_{i j}^{\mathfrak{A}}$, such that $f$ lifts via $\alpha$ and $\beta$ to the inclusion $\mathcal{B}_{t} \subseteq \mathcal{B}_{t+1}$. In turn there is a $\delta \in M$ with domain $\mathcal{A}_{j}$ such that $f$ lifts via $\gamma$ and $\delta$ to the inclusion $\mathcal{C}_{t} \subseteq \delta\left(\mathcal{A}_{j}\right)$. Set $\mathcal{C}_{t+1}=\operatorname{range}(\delta)$ and $\psi_{t+1}=\delta \circ \beta^{-1}$.

Now we define $\psi=\cup_{t} \psi_{t}$. Clearly $\psi$ has domain $\mathcal{B}$ and range $\subseteq \mathcal{S}$ containing $P$, and $\psi$ must be an embedding. To see that it is elementary, suppose that $\exists x \theta\left(\psi\left(b_{0}\right), \ldots, \psi\left(b_{s}\right), x\right)$ is an existential formula true in $\mathcal{S}$. Now $\psi_{2 s+2}=\gamma \circ \alpha^{-1}$ for some $\alpha \in M$ and $\gamma \in N$ with common domain $\mathcal{A}_{i} \in \mathfrak{A}$. Since $N$ is a correspondence system, there is a $\delta \in N$ (with some domain $\mathcal{A}_{j}$ ) and an $a \in \mathcal{A}_{j}$ and an $f \in I_{i j}^{\mathfrak{A}}$, such that $\theta\left(f\left(\alpha^{-1}\left(b_{0}\right)\right), \ldots, f\left(\alpha^{-1}\left(b_{s}\right)\right), a\right)$ holds in $\mathcal{A}_{j}$. But since $M$ is also a correspondence system, there is a $\beta \in M$ with the same domain $\mathcal{A}_{j}$ such that $f$ lifts to the inclusion $\mathcal{B}_{s} \subseteq \beta\left(\mathcal{A}_{j}\right)$ via $\alpha$ and $\beta$. Therefore $\theta\left(b_{0}, \ldots, b_{s}, \beta(a)\right)$ holds in $\mathcal{B}$. The dual argument shows that existential formulas 
true in $\mathcal{B}$ also hold in $\mathcal{S}$. But since existential formulas with parameters go back and forth between $\mathcal{B}$ and $\mathcal{S}$ this way, $\psi$ must be an elementary embedding.

Finally, given any $n$-type $\Gamma$ over any finite parameter set $P_{0} \subseteq P$, such that $\Gamma$ is realized in $\mathcal{S}$ by a tuple $\left(d_{1}, \ldots, d_{n}\right)$, we start with the substructure $\mathcal{P}_{0} \subseteq \mathcal{S}$ generated by $P_{0}$. Since $P_{0} \subseteq \operatorname{range}(\psi)$, we have a $t$ for which $P_{0} \subseteq \operatorname{range}\left(\psi_{t}\right)$. Let $\psi_{t}=\gamma \circ \alpha^{-1}$ with $\alpha \in M$ and $\gamma \in N$. There must be a $\delta \in N$ with some domain $\mathcal{A}_{j} \in \mathfrak{A}$ and an $f \in I_{i j}^{\mathfrak{A}}$ such that $f$ lifts via $\gamma$ and $\delta$ to the inclusion of $\mathcal{P}_{0}$ into the substructure generated by $\mathcal{P}_{0}$ and $d_{0}, \ldots, d_{n}$. But now there is also some $\beta \in M$ with domain $\mathcal{A}_{j}$ such that $f$ lifts via $\alpha$ and $\beta$ to the inclusion $\mathcal{B}_{t} \subseteq \beta\left(\mathcal{A}_{j}\right)$, and we set $b_{i}=\beta\left(\delta^{-1}\left(d_{i}\right)\right)$ and $c_{i}=\psi\left(b_{i}\right)$ for each $i$. Then $\left(c_{1}, \ldots, c_{n}\right)$ is an $n$-tuple within the image of $\psi$ which realizes the type $\Gamma$ over $P_{0}$, by standard arguments using $M$ and $N$.

When we have a parameter set $P$ as in Lemma 2, we refer to the image of $\mathcal{B}$ as a simulation of $\mathcal{S}$ over $P$. We might also refer to $\mathcal{B}$ itself the same way, but only when the embedding $\psi: \mathcal{B} \hookrightarrow \mathcal{S}$ is clear, because we need to know which elements $\psi^{-1}(p) \in \mathcal{B}$ correspond to the elements of $P$ in this simulation. Later we will discuss the extent to which $\mathcal{B}_{P}$ can be said to be uniform in $P$.

Corollary 32 Two countable structures with correspondence systems over the same uniformly computable cover are isomorphic.

Proof. Since $\mathcal{S}$ is countable, we simply set $P=\mathcal{S}$ and apply Lemma 2, whose proof may now be regarded as a back-and-forth construction of an isomorphism from $\mathcal{B}$ onto $\mathcal{S}$.

We are now ready for the main result of this section.

Theorem 33 Every perfectly locally computable structure $\mathcal{S}$ has a computable simulation $\mathcal{A}$, which can be embedded into $\mathcal{S}$ so as to simulate $\mathcal{S}$ over arbitrary countable parameter sets. Specifically, there is a set of elementary embeddings $\psi_{p}: \mathcal{A} \hookrightarrow \mathcal{S}$, one for each function $p: \omega \rightarrow \mathcal{S}$ which enumerates a countable parameter set $Q_{p}=\operatorname{range}(p) \subseteq \mathcal{S}$, such that:

- $Q_{p} \subseteq \psi_{p}(\mathcal{A}) ;$ and

- $\psi_{p}(\overline{\mathcal{A}})$ is a simulation of $\mathcal{S}$ over $Q_{p} ;$ and

- if $p$ and $p^{\prime}$ are two such functions and $p\left\lceil n=p^{\prime} \uparrow n\right.$, then for all $k<n$,

$$
\psi_{p}^{-1}(p(k))=\psi_{p^{\prime}}^{-1}\left(p^{\prime}(k)\right) .
$$

As a partial converse, every structure which has a computable simulation $\mathcal{A}$ with embeddings $\psi_{p}$ satisfying these conditions has a uniformly computable cover with a correspondence system.

To make this last claim an actual converse, we would need to show that the correspondence system for $\mathcal{S}$ is perfect. Whether this is true remains open. We also note that it would be equivalent to give the same statement only for finite parameter enumerations $p$, since the last condition would allow a simulation over a countable parameter set $P$ to be built by taking successive nested finite enumerations $p_{m} \subseteq p_{m+1}$ with $P=\cup_{m} \operatorname{range}\left(p_{m}\right)$, and setting $\psi=\lim _{m} \psi_{p_{m}}$ 
Proof. When we assume that $\mathcal{S}$ is perfectly locally computable, the existence of a computable simulation of $\mathcal{S}$ follows from Lemma 1, which also ensures that $\mathcal{S}$ and its computable simulation $\mathcal{A}$ both have perfect correspondence systems over the same uniformly computable cover. Therefore Lemma 2 shows that $\mathcal{A}$ can be elementarily embedded into $\mathcal{S}$ so as to simulate $\mathcal{S}$ over any parameter set $Q_{p}$ enumerated by a function $p: \omega \rightarrow \mathcal{S}$. Moreover, an examination of the proof of Lemma 2 shows that the embedding chooses the $a \in \mathcal{A}$ with $\psi_{p}(a)=p(k)$ using only the common cover $\mathfrak{A}$, its correspondence systems for $\mathcal{A}$ and $\mathcal{S}$, and the elements $p(0), p(1), \ldots, p(k)$ in $\mathcal{S}$. This proves the claim about parameter enumerations $p$ and $p^{\prime}$ which agree up to $n$.

The proof of the partial converse is longer and more technical, and we relegate it to Appendix A.

We can think of $\mathcal{B}_{P}$ as being built uniformly in the parameter set $P$ if the elements of $P$ are named as elements in different $\mathcal{A}_{i}$ in the cover $\mathfrak{A}$ of $\mathcal{S}$. That is, suppose that we are given a computable enumeration $\left\langle\left(i_{k}, a_{k}, f_{k}\right)\right\rangle_{k \in \omega}$ for which there exist maps $\beta_{k} \in N$ with $a_{k} \in \mathcal{A}_{i_{k}}=\operatorname{dom}\left(\beta_{k}\right)$ such that

- each $f_{k} \in \mathcal{I}_{i_{k}, i_{k+1}}^{\mathfrak{A}} ;$ and

$-\beta_{k+1} \circ f_{k}=\beta_{k} ;$ and

$-\left\{\beta_{k}\left(a_{k}\right): k \in \omega\right\}=P$.

Then we could build a computable copy of the simulation $\mathcal{B}_{P}$ of $\mathcal{S}$ over $P$, uniformly in the perfect cover of $\mathcal{S}$ and the enumeration $\left\langle\left(i_{k}, a_{k}, f_{k}\right)\right\rangle_{k \in \omega}$, and enumerate the image of $P$ in $\mathcal{B}_{P}$. More generally, if the enumeration $\left\langle\left(i_{k}, a_{k}, f_{k}\right)\right\rangle_{k \in \omega}$ has Turing degree $\boldsymbol{d}$, then with a $\boldsymbol{d}$-oracle we can build a copy of $\mathcal{B}_{P}$ in which the image of $P$ will be computably enumerable in $\boldsymbol{d}$. It is awkward to think of the set $P$ itself as having Turing degree $\boldsymbol{d}$, because an infinite set $P$ will have distinct enumerations with distinct Turing degrees, but within the cover $\mathfrak{A}$ of $\mathcal{S}$, we can view $P$ as being computably enumerable in $\boldsymbol{d}$, as well as in the degrees of other enumerations. Of course, $P$ itself, viewed as a subset of $\mathcal{S}$, does not admit effective enumeration in any obvious way.

\section{Category Theory}

In general, a cover of a structure $\mathcal{S}$ need not be a category. The cover has objects (the structures $\mathcal{A}_{i}$ ) and morphisms among them, but the definition does not require that the composition of two morphisms be a morphism, nor that the identity morphisms be included. Adding the identity morphisms would not be a problem, but it can happen that when one closes the sets of morphisms under composition, the resulting category is no longer a cover of $\mathcal{S}$, since the new morphisms may not correspond to any inclusion maps within $\mathcal{S}$. However, for perfectly locally computable structures, this difficulty vanishes.

Lemma 3. Let $\mathcal{S}$ be a perfectly locally computable structure. Then there is a perfect cover $\mathfrak{A}$ of $\mathcal{S}$, called the derived cover of $\mathcal{S}$, with the properties that every 
$I_{i i}^{\mathfrak{A}}$ contains the identity map on $\mathcal{A}_{i}$ and that for all $i, j, k \in \omega$ and all $f \in I_{i j}^{\mathfrak{A}}$ and $g \in I_{j k}^{\mathfrak{A}}$, we have $g \circ f \in I_{i k}^{\mathfrak{A}}$. Thus $\mathfrak{A}$ may be viewed as a computable category, with objects $\left\{\mathcal{A}_{i}: i \in \omega\right\}$ and with maps in $I_{i j}^{\mathfrak{A}}$ as the morphisms from $\mathcal{A}_{i}$ to $\mathcal{A}_{j}$.

To be careful, we should speak of the derived cover only when some perfect cover has already been specified.

Proof. $\mathfrak{A}$ is just the closure of the original perfect cover of $\mathcal{S}$ under the required properties. It is clear from Definition 23 that this $\mathfrak{A}$ is still a perfect cover of $\mathcal{S}$, and the uniform computability of the cover $\mathcal{A}$ follows from that of the original cover, since the underlying simple cover has not changed and the new sets $I_{i j}^{\mathfrak{A}}$ are derived from the old ones by existential conditions.

Category theorists have long known the category $\operatorname{FGSub}(\mathcal{S})$ of all finitely generated substructures of a structure $\mathcal{S}$, where the morphisms are just the inclusion maps within $\mathcal{S}$. Of course, if $\mathcal{S}$ is uncountable, then so is $\operatorname{FGSub}(\mathcal{S})$, so this presentation is not of immediate use for computability theorists. However, we do have a connection.

Proposition 41 If $\mathcal{S}$ is perfectly locally computable, then there exists a faithful functor $R$ mapping $\boldsymbol{F G S u b}(\mathcal{S})$ into the derived cover $\mathfrak{A}$ of $\mathcal{S}$. Moreover, there exists a natural isomorphism $\beta:\left(I_{\mathfrak{A}} \circ R\right) \rightarrow I_{F G S u b(\mathcal{S})}$ where the $I_{-}$denote the appropriate inclusions into the category of all $L$-structures under embeddings.

Proof. Let $\mathfrak{A}$ be a perfect cover of $\mathcal{S}$, with correspondence system $M$. For each $B \in \operatorname{FGSub}(\mathcal{S})$, fix $R(\mathcal{B})$ to be any $\mathcal{A}_{i} \in \mathfrak{A}$ which is the domain of some $\beta \in M$ whose image is $\mathcal{B} . \mathcal{A}_{i}$ and $\beta$ need not be unique, and it is startling that we can make such an arbitrary choice and have both a functor and a natural isomorphism, but the perfection of the cover allows it to work. Let $N \subseteq M$ contain exactly the maps $\beta$ chosen in this process. Thus each $\mathcal{B} \in \mathbf{F G S u b}(\mathcal{S})$ is the image of a unique $\beta_{\mathcal{B}} \in N$, whose domain is $R(\mathcal{B})$.

Now every morphism $\mathcal{B} \hookrightarrow \mathcal{C}$ in $\operatorname{FGSub}(\mathcal{S})$ is an inclusion $\mathcal{B} \subseteq \mathcal{C}$ within $\mathcal{S}$, and with $\beta_{\mathcal{B}} \in N$ as above, we have some $f \in I_{i j}^{\mathfrak{A}}$ for some $j$, and some $\gamma \in M$ with domain $\mathcal{A}_{j}$ and image $\mathcal{C}$, such that $\gamma \circ f=\beta_{\mathcal{B}}$. Now we also have some $\beta_{\mathcal{C}} \in M$ mapping $R(\mathcal{C})$ onto $\mathcal{C}$, say with $R(\mathcal{C})=\mathcal{A}_{k}$. Since $M$ is perfect, the map $\beta_{\mathcal{C}}^{-1} \circ \gamma$ must lie in $I_{j k}^{\mathfrak{A}}$. We then define $R$ to take the inclusion morphism $\mathcal{B} \subseteq \mathcal{C}$ to the morphism $R(\mathcal{B} \subseteq \mathcal{C})=\beta_{\mathcal{C}}^{-1} \circ \gamma \circ f$, which lies in $I_{i k}^{\mathfrak{A}}$, since $\mathfrak{A}$ is a derived cover. Again, an arbitrary choice of $f$ and $\gamma$ may have been involved here. However, for any $x \in R(\mathcal{B})=\mathcal{A}_{i}$, we have

$$
R(\mathcal{B} \subseteq \mathcal{C})(x)=\beta_{\mathcal{C}}^{-1} \circ \gamma \circ f(x)=\beta_{\mathcal{C}}^{-1} \circ \beta_{\mathcal{B}}(x),
$$

so that in fact the definition of $R(\mathcal{B} \subseteq \mathcal{C})$ was independent of this choice. Moreover, this will show that $R$ respects composition of morphisms, as functors must. Suppose that $\mathcal{B} \subseteq \mathcal{C} \subseteq \mathcal{D}$ are objects of $\operatorname{FGSub}(\mathcal{S})$. Then

$$
R(\mathcal{C} \subseteq \mathcal{D}) \circ R(\mathcal{B} \subseteq \mathcal{C})=\left(\beta_{\mathcal{D}}^{-1} \circ \beta_{\mathcal{C}}\right) \circ\left(\beta_{\mathcal{C}}^{-1} \circ \beta_{\mathcal{B}}\right)=\beta_{\mathcal{D}}^{-1} \circ \beta_{\mathcal{B}}=R(\mathcal{B} \subseteq \mathcal{D}) .
$$

Thus $R$ really is a functor.

The naturality of $\beta$ is evident from its construction. 
Corollary $42 \operatorname{colim}\left(I_{\mathfrak{A}} \circ R\right) \simeq \mathcal{S}$

Proof. Since $\beta$ is a natural isomorphism, we have $\operatorname{colim}\left(I_{\mathbf{F G S u b}(\mathcal{S})}\right) \simeq \operatorname{colim}\left(I_{\mathfrak{A}} \circ\right.$ $R)$. The former is easily seen to be $\mathcal{S}$.

The functor $R$ need not be onto. It is possible, for instance, for two distinct $\mathcal{A}_{i}$ and $\mathcal{A}_{j}$ in $\mathfrak{A}$ to map onto the same $\mathcal{B}$ via maps $\gamma, \delta \in M$, and also possible that these are the only maps in $M$ with domains $\mathcal{A}_{i}$ or $\mathcal{A}_{j}$. In such a case, one of $\mathcal{A}_{i}$ or $\mathcal{A}_{j}$ will not lie in the image of $R$. Of course, since $M$ is perfect, $\delta^{-1} \circ \gamma \in I_{i j}^{\mathfrak{A}}$ in this case, and this map is an isomorphism, as is $\gamma^{-1} \circ \delta \in I_{j i}^{\mathfrak{A}}$.

This can be done in a bit more canonically using $\beta$ as above:

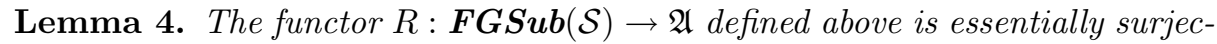
tive.

Proof. Fix any $\mathcal{A}_{i} \in \mathfrak{A}$. Then there is some $\alpha \in M$ with domain $\mathcal{A}_{i}$ and image $\mathcal{B}=\alpha\left(\mathcal{A}_{i}\right)$, and some $\beta_{\mathcal{B}} \in N \subseteq M$ with domain $\mathcal{A}_{j}=R(\mathcal{B})$ for some $j$. By perfection of $M, \beta_{\mathcal{B}}^{-1} \circ \alpha \in I_{i j}^{\mathfrak{A}}$, and this embedding maps $\mathcal{A}_{i}$ onto $R(\mathcal{B})$.

\section{References}

1. F. Borceux, Handbook of Categorical Algebra, 3 volumes (Cambridge University Press, 1994-1995).

2. S. Mac Lane, Categories for the Working Mathematician, 2nd edition (New York: Springer-Verlag, 1997).

3. R.G. Miller, Locally computable structures, in Computation and Logic in the Real World - Third Conference on Computability in Europe, CiE 2007, eds. B. Cooper, B. Löwe, \& A. Sorbi, Lecture Notes in Computer Science 4497 (Springer-Verlag: Berlin, 2007), 575-584. Also available at qcpages . qc . cuny . edu $\sim^{\sim}$ rmiller/research.

4. R.G. Miller, Local computability and uncountable structures, to appear.

5. R.I. Soare, Recursively Enumerable Sets and Degrees (New York: Springer-Verlag, 1987).

\section{Appendix A}

Here we complete the proof of Theorem 33 by proving the partial converse there stated. Suppose that $\mathcal{A}$ is a computable simulation of $\mathcal{S}$ and that there is a set of elementary embeddings $\psi_{p}: \mathcal{A} \hookrightarrow \mathcal{S}$, one for each function $p: \omega \rightarrow \mathcal{S}$ which enumerates a countable parameter set $Q_{p}=\operatorname{range}(p) \subseteq \mathcal{S}$, such that:

$-Q_{p} \subseteq \psi_{p}(\mathcal{A}) ;$ and

- $\psi_{p}(\mathcal{A})$ is a simulation of $\mathcal{S}$ over $Q_{p} ;$ and

- if $p$ and $p^{\prime}$ are two such functions and $p\left\lceil n=p^{\prime}\lceil n\right.$, then for all $k<n$,

$$
\psi_{p}^{-1}(p(k))=\psi_{p^{\prime}}^{-1}\left(p^{\prime}(k)\right) .
$$


We have to show that the existence of such an $A$ implies that $\mathcal{S}$ has a uniformly computable cover with a correspondence system. Now $\mathcal{A}$ has a perfect cover $\mathfrak{A}$, by Theorem 24 . Let $M$ be a perfect correspondence system for $\mathcal{A}$ and $\mathfrak{A}$. The correspondence system $N$ will consist of all maps of the form $\psi_{p} \circ \alpha$, for all finite $p: n \rightarrow \mathcal{S}$ and all $\alpha \in M$ such that range( $\alpha)$ is generated by $\left\{\psi_{p}^{-1}(p(i)): i<n\right\}$. (Here we think of a finite function $p: n \rightarrow \mathcal{S}$ as a function from $\omega$ into $\mathcal{S}$ by repeating its image over and over: $p(k+n m)=p(k)$ for all $k$ and $m$.)

Now each finitely generated $\mathcal{C} \subseteq \mathcal{S}$ with generators enumerated by $p$ lies within the image of $\psi_{p}$, and the finitely generated substructure $\psi_{p}^{-1}(\mathcal{C}) \subseteq \mathcal{A}$ must be the image of some $\mathcal{A}_{i} \in \mathfrak{A}$ under some $\alpha \in M$, since $M$ is a perfect cover of $\mathcal{A}$. Hence $\mathcal{C}=\left(\psi_{p} \circ \alpha\right)\left(\mathcal{A}_{i}\right)$ is the image of some map in $N$. Likewise, each $\mathcal{A}_{i} \in \mathfrak{A}$ is the domain of some $\alpha \in M$, hence also of some map in $N$. Moreover, each $f \in I_{i j}^{\mathfrak{A}}$, for any $i$ and $j$, lifts to an inclusion map within $\mathcal{A}$, and then lifts further to an inclusion map within $\mathcal{S}$, via any $\psi_{p}$ we like. Conversely, any inclusion $\mathcal{C}^{\prime} \subseteq \mathcal{C}$ of finitely-generated substructures of $\mathcal{S}$ is the lift (via $\psi_{p}$, where $p$ enumerates first the generators of $\mathcal{C}^{\prime}$, and then the generators of $\mathcal{C}$ ) of an inclusion in $\mathcal{A}$, which in turn is the lift of some $f$ in some $I_{i j}^{\mathfrak{A}}$ via some $\alpha, \beta \in M$. If $p^{\prime}$ is the restriction of $p$ to the generators of $\mathcal{C}^{\prime}$, then the inclusion $\mathcal{C}^{\prime} \subseteq \mathcal{C}$ is the lift of $f$ via $\left(\psi_{p^{\prime}} \circ \alpha\right)$ and $\left(\psi_{p} \circ \beta\right)$, which both lie in $N$. Thus $\mathfrak{A}$ is a uniformly computable cover of $\mathcal{S}$.

The preceding remarks also proved the first three parts in Definition 23. For part 4 , fix any $f \in I_{i j}^{\mathfrak{A}}$ for any $i$ and $j$, along with any $\beta \in N$ with domain $\mathcal{A}_{i}$. Then $\beta=\psi_{p} \circ \alpha$ for some $\alpha \in M$ and some $p: n \rightarrow \mathcal{S}$ for which $\left\{\psi_{p}^{-1}(p(k): k<\right.$ $n\}$ generates range $(\alpha)$. Since $M$ is a correspondence system, there is a $\gamma \in M$ with domain $\mathcal{A}_{j}$ such that $\alpha=\gamma \circ f$. But now there is a finite $q$ such that $q\left\lceil n=p\right.$ and $q(n+k)=\psi_{p}\left(a_{k}\right)$, where $a_{0}, \ldots, a_{m}$ generate $\gamma\left(\mathcal{A}_{j}\right)$ within $\mathcal{A}$. So $\left(\psi_{q} \circ \gamma\right) \in N$, and

$$
\left(\psi_{q} \circ \gamma \circ f\right)=\left(\psi_{q} \circ \alpha\right)=\left(\psi_{p} \circ \alpha\right),
$$

with the last equality following because $p\lceil n=q\lceil n$ and range $(\alpha)$ is generated by the elements $\psi_{p}^{-1}(p(k))=\psi_{q}^{-1}(q(k))$ for $k<n$. This proves part 4 .

For part 5 of Definition 23, fix any $\beta \in N$ with domain $\mathcal{A}_{i}$ and any finitely generated $\mathcal{C} \subseteq \mathcal{S}$ with $\beta\left(\mathcal{A}_{i}\right) \subseteq \mathcal{C}$. Now $\beta=\psi_{p} \circ \alpha$ for some $\alpha \in M$ and some finite $p: n \rightarrow \mathcal{S}$, with the elements $\psi_{p}^{-1}(p(k))$ generating range $(\alpha)$. Let $q(k)=p(k)$ for $k<n$, and let $q(n), \ldots, q(n+m-1)$ enumerate the generators of $\mathcal{C}$ in $\mathcal{S}$. By assumption, $\psi_{q}$ is an elementary embedding of $\mathcal{A}$ into $\mathcal{S}$ whose image contains range $(q)$. Let $\mathcal{D}=\left\langle\psi_{q}^{-1}(q(k)): k<m\right\rangle \subseteq \mathcal{A}$. Since $\psi_{q}^{-1}(q(k))=\psi_{p}^{-1}(p(k))$ for all $k<n$, we know that $\alpha\left(\mathcal{A}_{i}\right) \subseteq \mathcal{D}$, and since $M$ is a correspondence system, there is some $\beta \in M$ and some $j$ and $f \in I_{i j}^{\mathfrak{A}}$ with $\mathcal{D}=\operatorname{range}(\beta)$ and $\beta \circ f=\alpha$. But then

$$
\left(\psi_{q} \circ \beta \circ f\right)=\left(\psi_{q} \circ \alpha\right)=\left(\psi_{p} \circ \alpha\right),
$$

proving part 5 of Definition 23, since $\left(\psi_{q} \circ \beta\right) \in N$. Thus $\mathcal{A}$ is a uniformly computable cover of $\mathcal{S}$ with correspondence system $N$. 